\title{
How to Commit Companies in Collaborative Empirical Research
}

\author{
Markku Oivo \\ M3S Research Unit \\ University of Oulu \\ Oulu, Finland \\ Markku.Oivo@oulu.fi
}

\begin{abstract}
Getting companies involved in and committed to empirical research projects is a major challenge for most researchers. This paper discusses several cases of lessons learned about how to involve and get the commitment of companies in different types of empirical research projects. This paper will discuss the experiences of two very large consortium projects and one medium-size research project. In addition, the roles of meetings, workshops, and off-site meetings are also discussed.
\end{abstract}

\section{(Abstract)}

Keywords: empirical software engineering, experiments, case studies, need for speed, N4S, cloud software, ESEIL, FiDiPro, Digile, ICT SHOK, public-private partnership, PPP.

\section{INTRODUCTION}

The literature contains plenty of guidelines for carrying out empirical software engineering research. Typically, these guidelines take the researcher's perspective and give advice on how to carry out experiments, case studies, surveys, etc. However, most of them implicitly or explicitly assume that companies are somehow magically recruited, involved in, and committed to the empirical research process. Advice on how to recruit companies in the first place and, more importantly, how to get them truly committed to the process are scarce.

One of the major challenges of empirical software engineering is finding industrial partners for case studies and experiments. Many companies have carried our major cost-saving activities and their R\&D resources are tightly focused on strategic product development with very little resources available for anything but near-term or short-term product roadmaps.
This paper discusses challenges and potential solutions when selecting companies for empirical software engineering research, how to get selected companies interested, how to encourage their commitment, and finally how to involve them in the research. We will focus on case studies and experiments. This paper is based on personal experience, including 35 years in $R \& D$ in academia, industry, and applied research institutes in six different countries. The lessons learned are based on several EU projects in FP programs, ITEA, Horizon 2020, very large industryacademia consortium projects consisting of dozens of partners, medium-size research projects involving one research partner and one to five companies, small-scale $\mathrm{PhD}$ and $\mathrm{MSc}$ thesis research projects with companies, as well as surveys at the global, national and company levels.

This paper will also present practical examples from three applied research projects. The first two are large national consortium projects. The first of these projects is Cloud Software (2010-2013), a large consortium project focusing on cloud, lean, and agile software development. The second project is Need for Speed (N4S), a very large national research program with over 30 industrial partners and 10 research partners. The third project is ESEIL, a research project focusing on industrial experiments carried out by one research partner with five companies.

\section{PRECONDITIONS FOR INVOLVING COMPANIES IN CASE STUDIES AND EXPERIMENTS}

Getting companies interested in research is, in many ways, similar to business-to-business sales, especially in the consulting business context. 
Researchers typically have spent a lot of time investigating their research topics and have grown to be dedicated experts in their specific topics. It is very tempting and natural to approach a potential partner company with a "product first" strategy and to make a "take it or leave it" offer. However, thorough discussions and meetings with companies are required in order to understand their business and determine their needs and priorities.

Modern theories of value sales can be applied to marketing research offerings. Researchers should familiarize themselves with the business and technology of the potential partner company and create a value offering that explains how the research will bring value to the company. Ideally, the researcher should be able to build a value cocreation situation with the company and research organization. The door-to-door salesman approach where a researcher tries to sell a fixed (research) product to an unknown customer is seldom successful.

Researchers should familiarize themselves with the companies' challenges in the business environment and try to match their research with the issues that the companies are trying to improve. If this turns out to be very difficult, it is often a sign that there is not enough common interest to go forward with the research proposal. In this case, it may make sense to look to the future and discuss whether this topic could be of interest in the coming years.

A "match made in heaven" occurs when a company is already carrying out research or improvements in the area of the research proposal. Based on the experience of being involved in negotiations on both sides of the table, a practical piece of advice is that companies should not join an empirical study if they do not plan to work on this topic anyway. Otherwise, it will be very difficult to invest the time in the study and it will remain a low priority.

However, when research topic is in the agenda of the company, it becomes easy to invest time (which is more precious than money) and the company will get help from researchers who are leading edge experts of their fields. It becomes a win-win situation for researchers and companies.

The benefits are even greater when multiple case studies and replicated experiments are conducted with several companies at the same time. This gives companies an opportunity to share lessons learned and best practices. This kind of collaboration among the companies has turned out to be extremely valuable in consortia projects, such as Cloud Software and N4S.

\section{PRACTICAL EXAMPLES}

\section{A. Tekes and ICT SHOK}

Finland has a long tradition of industryacademia collaboration, which started already in 1980's with the establishment of the Tekes national funding organization in 1983. Tekes funding has traditionally focused both on research in academia and supporting $R \& D$ in companies.

Tekes strongly encourages and even requires collaboration between large companies, small- and medium-sized enterprises (SME), and research organizations. Large companies are also required to collaborate with SMEs and academics. Likewise, funding for research organizations has typically required close collaboration with industry.

In 2006, the Finnish Research and Innovation Council made a radical shift in research funding and decided to establish the so-called Strategic Centres for Science, Technology, and Innovation (SHOK). The objective was to develop and apply new methods for cooperation, co-creation, and interaction.

The SHOKs started in 2008 and were organized as seven companies owned by private companies and research organizations. SHOKs received major funding from Tekes and channeled it to joint industry-academia research programs. The total volume of SHOK research funding in 2011 was $189 \mathrm{M} €$. 


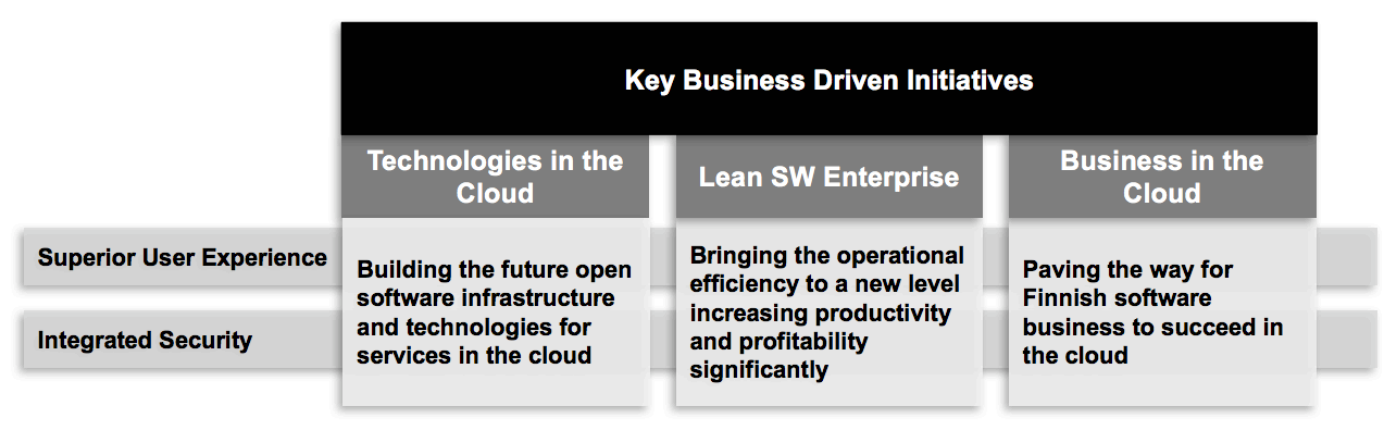

Figure 1. Cloud Software program.

Research organizations typically received $70 \%$ funding of their expenses (full cost model) and companies received 30-50\% funding of their expenses. The Academy of Finland supports SHOKs with funding for researchers and research organizations.

Digile Ltd. (originally named Tivit) was established as one of the seven SHOK companies and focused on ICT research and development (R\&D). In 2012, Digile research programs provided 43 M€ in funding for 102 companies and 12 research organizations. The programs typically lasted two to four years. However, part of cost saving operations, the Finnish government made the controversial decision in 2015 to cut research funding and gradually terminate the special funding to SHOK companies.

Digile executed two major programs related to software engineering: Cloud Software (2010-2013) and N4S (2014-2017). The following chapters describe the experiences from these two programs.

\section{B. Cloud Software}

Digile (Tivit at the time) established a large Cloud Software research program in 2010 that included 20 companies and eight research organizations. With a total budget of $60 \mathrm{M} €$, the program claimed to have achieved $100 \mathrm{M} €$ annual benefits for the companies.

Encouraging companies and research organizations to work closely together was a key objective of the program. A strategic research agenda (SRA) was developed before the launch of the program and published in 2010. The future scenario of the SRA reached into 2017. Creating the SRA was a collaborative effort between the research organizations and companies and laid the foundation for getting the companies involved in the program.

Quick business benefits were important targets of the research program. Therefore, the project used the novel method of three-month research sprints, which introduced an agile way of working into the process. This agile research process was further developed in the N4S program.

Traditionally, externally funded research projects are carefully planned during the proposal phase and the implementation has been expected to follow the plan. Cloud Software program took a different approach by emphasizing iterative planning and sharing during the research process. This approach has similarities with agile methods but has naturally longer cycles than agile software development.

The plans were updated every three months, and the results and plans were checked and updated during quarterly consortium review meetings. The emphasis was on working together and learning together. All of the partners participated in the quarterly meetings, which typically lasted for two days. The quarterly meetings evolved into effective collaboration tools that involved heavy interaction between research and companies. The quarterly meetings typically involved several hands-on workshops where researchers and companies worked together.

\section{NEED FOR SPEED}

This chapter describes the N4S program (http:/www.n4s.fi/en/) as an example of empirical research in industry with a large research consortium [1]. The project started in 2014 and will end in May 2017. 


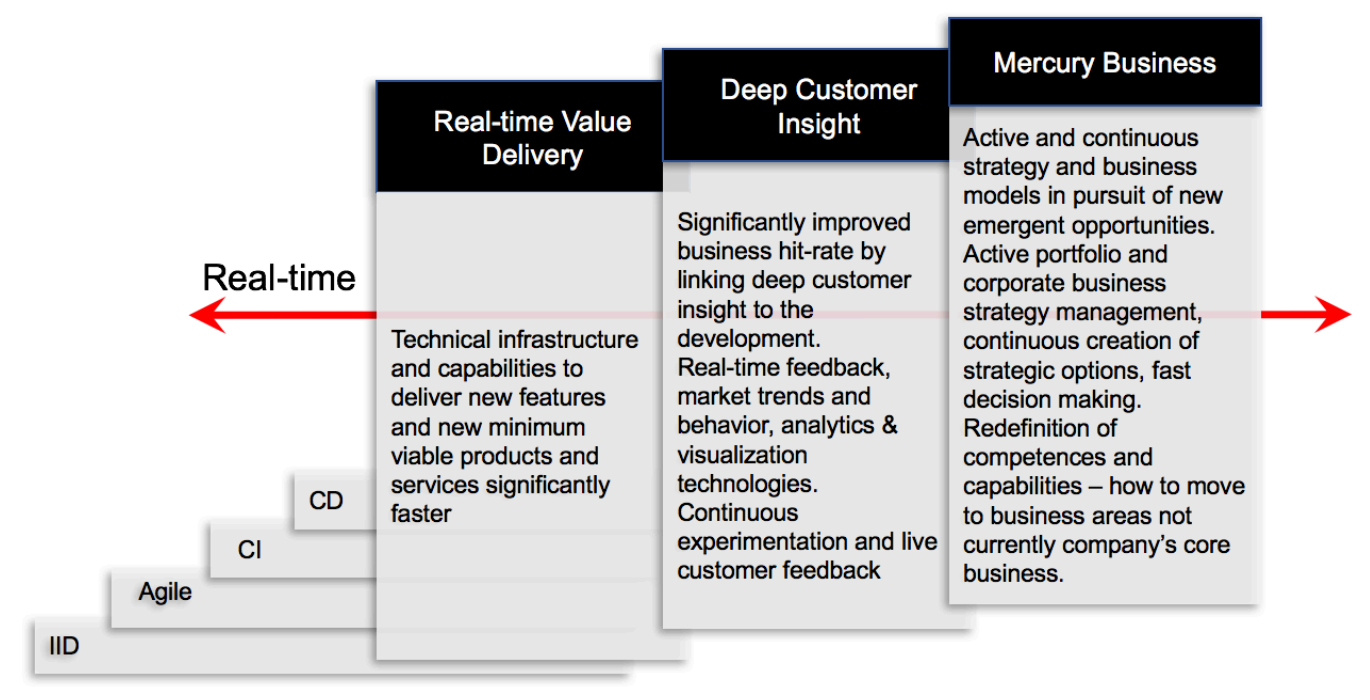

Figure 2. Need for Speed program.

\section{A. Background}

The success of the Cloud Software program laid the foundation for the preparation of N4S program even though $\mathrm{N} 4 \mathrm{~S}$ was in no means a continuation of the Cloud Software program. A core team of 17 experts, many of which worked in the Cloud Software consortium, led the preparations and writing of the new SRA. The preparation went smoothly, partly because many of the members knew each other well from the Cloud Software program.

\section{B. Selecting and recruiting companies for N4S}

A large series of open workshops were organized in three cities (Helsinki, Oulu, and Tampere) and used World Café (http://www.theworldcafe.com) for the scenario work. The results from the workshops were integrated into the SRA by a core team of authors. In total, over 200 experts from over 60 organizations participated in the preparation of the SRA. Seventeen research organizations participated in the work; therefore, the majority of the organizations were from industry.

This wide participation ensured that industry interests and relevant topics were addressed in the SRA. It also contributed to information sharing and dissemination of the SRA to a wide industry audience. As a result, the final consortium included leading-edge Finnish companies that were working in the area of the project.

\section{Company commitment: research collaboration in action}

N4S took a fairly radical approach to a research strategy and planning process that was driven by business cases. All of the companies were required to create business cases for the topics that they wanted to be included in their research. They were not allowed to work alone and they had to have research partners in their business cases. Likewise, all research partners were required to link their research to one or more of the business cases. No research was approved without a link to a business case.

Most partners accepted this bi-directional push toward collaboration between industry and research positively. It pushed the researchers to search for the industrial relevance of their research in advance and pushed industry partners to actively collaborate with research partners in empirical research in real industrial environments.

N4S adopted the agile project management principles developed during the Cloud Software program. Every three months, the whole 
consortium had a joint two-day meeting, which included several types of plenary sessions and workshops. The aim of the meetings was to review the results of the past quarter and plan for the next quarter.

These quarterly review meetings included plenary presentations, world café-type workshops, research bazaars, hackathons, and many other types of innovative collaboration events. The results from the past quarter were presented with demos, poster, presentations, etc. Every partner was expected to participate in these quarterly meetings.

N4S took a business oriented approach also in the composition of the steering group, which was responsible for the management decisions of the of the program. The steering group consists of mainly of industrial partners. Extended steering group meetings were held during the quarterly review meetings and included also research partners. From a research perspective, this was new and a bit awkward, but from the viewpoint of involving companies in the research, it was a valuable approach.

\section{THE EXPERIMENTAL SOFTWARE ENGINEERING INDUSTRIAL LABORATORY}

The aim of the Experimental Software Engineering Industrial Laboratory (ESEIL http://www.softwareindustryexperiments.org) project is to perform controlled experiments in industry to analyze processes and methods used in professional software development in different environments [2], [3]. The project, which started in 2012 and will end in 2017, is being conducted by M3S University of Oulu with five industrial partners. The research work is funded by Tekes and in part by the five companies. The project belongs to the Finnish Distinguished Professor program (FiDiPro professor Natalia Juristo).

The goals of ESEIL are two-fold: (1) to improve the quality of software built in industry and (2) to advances the state of the knowledge on software engineering controlled experiments. It will also help organizations to identify and control about the variables that influence their software development processes in order to improve their processes and make them more predictable.
ESEIL uses two approaches to encourage the recruitment and commitment of companies in empirical research. The first is based on ESEIL's goal to improve the operational efficiency of the participating companies as soon as possible during the research project. This provides a crucial financial incentive for companies to invest valuable time in the experiment. Another approach is to provide free training on the method (TDD) to be experimented in the company. The experiments have been embedded in the training course. This has been useful for recruiting company participants in experiments. However, the success of the second strategy depends on the culture of the company: companies that regularly invest in training are naturally easier to recruit.

\section{OULANKA OFF-SITE}

Empirical research in industry is very human oriented. It requires trust and close collaboration between researchers and companies. This differs significantly from more technical and theoretical computer science. Networking is a big benefit and helps in the recruitment of companies and working in the empirical studies. An effective way of fostering networking is to put people in the same isolated space for more than a few hours.

The Oulanka biological research station (http://www.oulu.fi/oulankaresearchstation/) is in the wilderness in the Northeastern part of Finland. The M3S research unit at the University of Oulu has used Oulanka to host numerous software engineering research meetings both in the project preparation phase and also to host project meetings. It has turned out to be an excellent location for off-site meetings. It has hosted several Cloud Software and N4S consortium meetings.

The unique national park surrounding the station provides a stimulating environment. The remote location creates and environment of little distractions from the work. Moreover, combining intensive workshops, nature, and sauna helps people to socialize and focus on intensive collaboration. Researchers learn the challenges of the companies and start thinking about how to create value for the companies through research.

Oulanka has turned out to be a very effective tool for networking between researchers and companies. It helps to create trust among 
participants and creates an environment that boosts the productivity of meetings.

\section{CONCLUSIONS}

This paper discusses success factors for getting companies involved in joint research collaboration during empirical studies. Companies should be involved heavily in the planning of the studies. The more business driven the planning is, the easier it will be to get companies involved in the empirical research. Companies live with much shorter planning cycles than traditional research projects that span two to five years. Having a more agile planning and management process (e.g., quarterly reviews and planning) helps to keep companies interested during the research process. The participation of industry representatives in the steering (group) of a research projects also helps to ensure that the research is industry relevant. Providing measurable business benefits with improved processes and practices is also a significant motivator.
However, these benefits come with a price: it can be challenging to stay focused on the longterm research agenda while maintaining industry interest in a multi-year research project.

\section{ACKNOWLEDGMENTS}

Tekes, Digile, DIMECC, M3S, and all the N4S partners are gratefully acknowledged for their collaboration during the research projects.

\section{REFERENCES}

[1] Järvinen, J., Huomo, T., Mikkonen, T., \& Tyrväinen, P. (2014, June). From agile software development to mercury business. In International Conference of Software Business (pp. 58-71). Springer International Publishing.

[2] Natalia Juristo, N. (2016). Experiences conducting experiments in industry: the ESEIL FiDiPro project. In Proceedings of the 4th International Workshop on Conducting Empirical Studies in Industry (CESI '16). ACM, New York, NY, USA, 1-3. DOI: https://doi.org/10.1145/2896839.2896846

[3] Fucci, D., Turhan, B., Juristo, N., Dieste, O., Tosun-Misirli, A., \& Oivo, M. (2015). Towards an operationalization of test-driven development skills: An industrial empirical study. Information and Software Technology, 68, 82-97. 